\title{
Evaluation of salt bridge structure and energetics in peptides using explicit, implicit and hybrid solvation models
}

\author{
Asim Okur, Lauren Wickstrom and Carlos Simmerling
}

Supplementary Material 
Table S.1. Temperature distributions and observed exchange ratios for TIP3P, GB ${ }^{\mathrm{OBC}}$ and Hybrid REMD simulations

\begin{tabular}{|c|c|c|c|c|c|}
\hline $\begin{array}{l}\text { Replica } \\
\text { Number } \\
\end{array}$ & $\begin{array}{c}\text { Tip3p } \\
\text { Temperature } \\
(\mathrm{K}) \\
\end{array}$ & $\begin{array}{c}\text { Exchange } \\
\text { Ratio }\end{array}$ & $\begin{array}{l}\text { Replica } \\
\text { Number } \\
\end{array}$ & $\begin{array}{c}\text { GB } \\
\begin{array}{c}\text { Temperature } \\
(\mathbf{K})\end{array} \\
\end{array}$ & $\begin{array}{c}\text { Exchange } \\
\text { Ratio } \\
\end{array}$ \\
\hline 1 & 295.5 & 0.08 & 1 & 300.0 & 0.12 \\
\hline 2 & 300.0 & 0.17 & 2 & 348.7 & 0.24 \\
\hline 3 & 304.6 & 0.17 & 3 & 405.3 & 0.23 \\
\hline 4 & 309.2 & 0.17 & 4 & 471.0 & 0.23 \\
\hline 5 & 313.9 & 0.18 & 5 & 547.5 & 0.25 \\
\hline 6 & 318.7 & 0.18 & 6 & 636.3 & 0.13 \\
\hline 7 & 323.6 & 0.18 & & & \\
\hline 8 & 328.5 & 0.18 & & & \\
\hline 9 & 333.6 & 0.19 & & Hybrid & \\
\hline 10 & 338.6 & 0.20 & $\begin{array}{l}\text { Replica } \\
\text { Number } \\
\end{array}$ & $\begin{array}{c}\text { Temperature } \\
\text { (K) } \\
\end{array}$ & $\begin{array}{c}\text { Exchange } \\
\text { Ratio } \\
\end{array}$ \\
\hline 11 & 343.8 & 0.20 & 1 & 269.5 & 0.08 \\
\hline 12 & 349.1 & 0.20 & 2 & 300.0 & 0.17 \\
\hline 13 & 354.4 & 0.21 & 3 & 334.0 & 0.19 \\
\hline 14 & 359.8 & 0.21 & 4 & 371.8 & 0.21 \\
\hline 15 & 365.3 & 0.21 & 5 & 413.9 & 0.23 \\
\hline 16 & 370.9 & 0.22 & 6 & 460.7 & 0.24 \\
\hline 17 & 376.5 & 0.23 & 7 & 512.9 & 0.26 \\
\hline 18 & 382.3 & 0.23 & 8 & 570.9 & 0.13 \\
\hline 19 & 388.1 & 0.24 & & & \\
\hline 20 & 394.0 & 0.24 & & & \\
\hline 21 & 400.0 & 0.25 & & & \\
\hline 22 & 406.1 & 0.25 & & & \\
\hline 23 & 412.3 & 0.26 & & & \\
\hline 24 & 418.6 & 0.26 & & & \\
\hline 25 & 425.0 & 0.27 & & & \\
\hline 26 & 431.5 & 0.27 & & & \\
\hline 27 & 438.1 & 0.27 & & & \\
\hline 28 & 444.8 & 0.28 & & & \\
\hline 29 & 451.5 & 0.29 & & & \\
\hline 30 & 458.4 & 0.29 & & & \\
\hline 31 & 465.4 & 0.29 & & & \\
\hline 32 & 472.5 & 0.30 & & & \\
\hline 33 & 479.7 & 0.30 & & & \\
\hline 34 & 487.1 & 0.31 & & & \\
\hline 35 & 494.5 & 0.31 & & & \\
\hline 36 & 502.0 & 0.31 & & & \\
\hline 37 & 509.7 & 0.32 & & & \\
\hline 38 & 517.5 & 0.33 & & & \\
\hline 39 & 525.4 & 0.33 & & & \\
\hline 40 & 533.4 & 0.34 & & & \\
\hline 41 & 541.5 & 0.34 & & & \\
\hline 42 & 549.8 & 0.34 & & & \\
\hline
\end{tabular}




$\begin{array}{lll}43 & 558.2 & 0.35 \\ 44 & 566.7 & 0.35 \\ 45 & 575.4 & 0.36 \\ 46 & 584.1 & 0.18\end{array}$

Table S.2. The populations and average errors for the clusters shown in Figure 2.

\begin{tabular}{|c|c|c|c|c|c|c|}
\hline \multirow[b]{3}{*}{ Number } & \multicolumn{6}{|c|}{ Backbone cluster populations } \\
\hline & \multicolumn{2}{|c|}{ TIP3P } & \multicolumn{2}{|c|}{ GB } & \multicolumn{2}{|c|}{ Hybrid } \\
\hline & Population & Error & Population & Error & Population & Error \\
\hline 1 & 6.21 & 4.10 & 5.06 & 0.41 & 5.56 & 3.05 \\
\hline 2 & 20.30 & 7.53 & 8.78 & 3.10 & 12.98 & 3.69 \\
\hline 3 & 7.05 & 3.42 & 58.92 & 9.12 & 10.33 & 2.20 \\
\hline 4 & 0.24 & 0.16 & 0.50 & 0.20 & 0.58 & 0.15 \\
\hline 5 & 1.98 & 0.59 & 5.62 & 0.77 & 3.20 & 1.54 \\
\hline 6 & 1.70 & 0.79 & 0.27 & 0.11 & 1.30 & 0.70 \\
\hline 7 & 10.28 & 4.60 & 2.01 & 0.92 & 5.49 & 0.59 \\
\hline 8 & 1.41 & 0.78 & 0.94 & 0.04 & 1.81 & 0.85 \\
\hline 9 & 0.76 & 0.28 & 4.44 & 0.60 & 0.88 & 0.62 \\
\hline 10 & 5.48 & 1.74 & 1.05 & 0.27 & 3.04 & 1.29 \\
\hline 11 & 0.50 & 0.09 & 0.26 & 0.05 & 0.36 & 0.04 \\
\hline 12 & 1.42 & 1.15 & 0.47 & 0.10 & 1.23 & 0.75 \\
\hline 13 & 2.96 & 1.01 & 1.04 & 0.23 & 5.47 & 2.82 \\
\hline 14 & 17.21 & 5.85 & 1.46 & 1.06 & 14.54 & 5.74 \\
\hline 15 & 3.48 & 0.30 & 0.51 & 0.18 & 4.38 & 2.73 \\
\hline 16 & 0.56 & 0.38 & 0.18 & 0.09 & 0.52 & 0.29 \\
\hline 17 & 0.10 & 0.04 & 0.14 & 0.01 & 0.52 & 0.12 \\
\hline 18 & 4.11 & 1.31 & 0.26 & 0.08 & 3.65 & 1.28 \\
\hline 19 & 0.28 & 0.25 & 1.16 & 0.19 & 0.66 & 0.47 \\
\hline 20 & 0.16 & 0.00 & 0.06 & 0.06 & 1.20 & 0.10 \\
\hline 21 & 1.30 & 0.63 & 1.50 & 0.05 & 0.84 & 0.36 \\
\hline 22 & 0.67 & 0.33 & 0.44 & 0.15 & 1.22 & 0.10 \\
\hline 23 & 0.01 & 0.01 & 0.05 & 0.02 & 0.01 & 0.01 \\
\hline 24 & 2.05 & 0.87 & 0.71 & 0.18 & 2.09 & 0.86 \\
\hline 25 & 0.54 & 0.32 & 1.20 & 0.24 & 0.91 & 0.27 \\
\hline 26 & 0.04 & 0.04 & 0.44 & 0.08 & 0.42 & 0.12 \\
\hline 27 & 2.29 & 0.38 & 0.28 & 0.04 & 1.60 & 0.92 \\
\hline 28 & 0.05 & 0.02 & 0.02 & 0.01 & 0.09 & 0.08 \\
\hline 29 & 0.40 & 0.12 & 0.08 & 0.00 & 0.21 & 0.04 \\
\hline 30 & 0.40 & 0.26 & 0.08 & 0.01 & 0.52 & 0.22 \\
\hline 31 & 0.50 & 0.18 & 0.13 & 0.05 & 0.45 & 0.16 \\
\hline 32 & 0.01 & 0.01 & 0.01 & 0.00 & 0.00 & 0.00 \\
\hline 33 & 0.00 & 0.00 & 0.09 & 0.09 & 0.09 & 0.09 \\
\hline 34 & 0.00 & 0.00 & 0.04 & 0.04 & 0.17 & 0.12 \\
\hline 35 & 0.41 & 0.18 & 0.04 & 0.01 & 0.48 & 0.12 \\
\hline
\end{tabular}




$\begin{array}{lllllll}36 & 0.00 & 0.00 & 0.32 & 0.31 & 1.74 & 0.60 \\ 37 & 0.03 & 0.03 & 0.11 & 0.11 & 1.16 & 0.62 \\ 38 & 0.77 & 0.77 & 0.01 & 0.01 & 2.95 & 2.37 \\ 39 & 0.01 & 0.01 & 0.05 & 0.02 & 0.16 & 0.13 \\ 40 & 0.79 & 0.33 & 0.06 & 0.00 & 1.01 & 0.92 \\ 41 & 0.00 & 0.00 & 0.10 & 0.10 & 0.00 & 0.00 \\ 42 & 0.22 & 0.22 & 0.08 & 0.00 & 0.62 & 0.41 \\ 43 & 0.13 & 0.13 & 0.00 & 0.00 & 0.24 & 0.24 \\ 44 & 0.07 & 0.02 & 0.01 & 0.01 & 0.12 & 0.05 \\ 45 & 0.00 & 0.00 & 0.00 & 0.00 & 0.07 & 0.07 \\ 46 & 0.04 & 0.03 & 0.00 & 0.00 & 0.29 & 0.06 \\ 47 & 0.22 & 0.22 & 0.00 & 0.00 & 0.03 & 0.00 \\ 48 & 0.36 & 0.32 & 0.42 & 0.02 & 0.71 & 0.60 \\ 49 & 0.04 & 0.01 & 0.00 & 0.00 & 0.33 & 0.27 \\ 50 & 0.98 & 0.00 & 0.18 & 0.04 & 0.70 & 0.33 \\ 51 & 0.10 & 0.04 & 0.02 & 0.02 & 0.16 & 0.07 \\ 52 & 0.31 & 0.18 & 0.09 & 0.04 & 0.62 & 0.44 \\ 53 & 0.00 & 0.00 & 0.00 & 0.00 & 0.05 & 0.05 \\ 54 & 0.12 & 0.08 & 0.02 & 0.02 & 0.16 & 0.13 \\ 55 & 0.12 & 0.09 & 0.01 & 0.01 & 0.39 & 0.20 \\ 56 & 0.00 & 0.00 & 0.00 & 0.00 & 0.04 & 0.04 \\ 57 & 0.01 & 0.01 & 0.00 & 0.00 & 0.05 & 0.05 \\ 58 & 0.12 & 0.08 & 0.02 & 0.01 & 0.19 & 0.08 \\ 59 & 0.05 & 0.04 & 0.04 & 0.01 & 0.12 & 0.10 \\ 60 & 0.00 & 0.00 & 0.00 & 0.00 & 0.27 & 0.27 \\ 61 & 0.01 & 0.00 & 0.01 & 0.01 & 0.20 & 0.16 \\ 62 & 0.25 & 0.15 & 0.03 & 0.00 & 0.18 & 0.04 \\ 63 & 0.12 & 0.07 & 0.00 & 0.00 & 0.05 & 0.05\end{array}$




\section{Saltbridge TIP3P REMD}

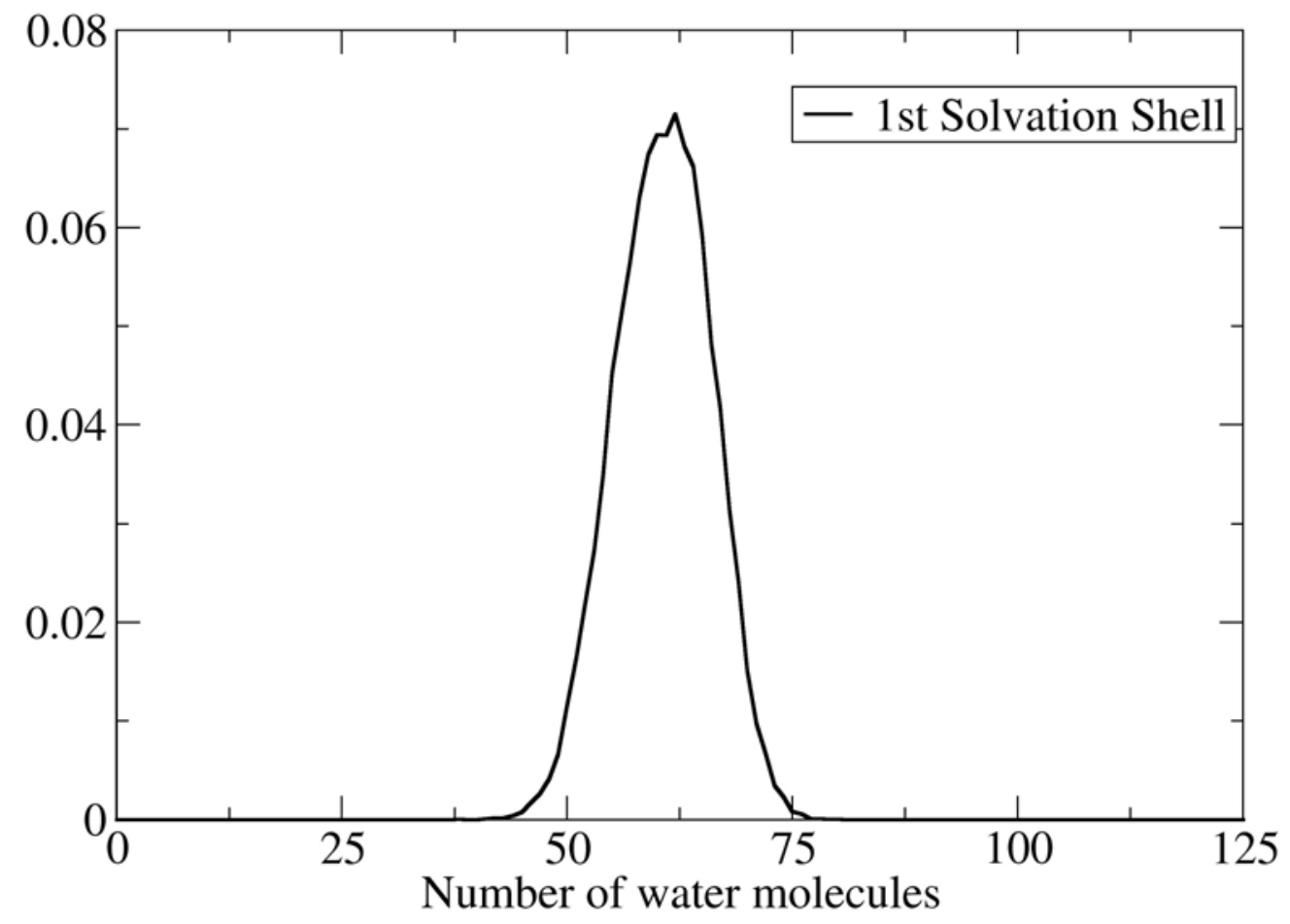

Figure S.1. Distributions of water molecules within the first solvent shell calculated using the $300 \mathrm{~K}$ trajectory from fully solvated REMD simulations. 75 water molecules are enough to solvate the first shell for every conformation observed in REMD simulations. 


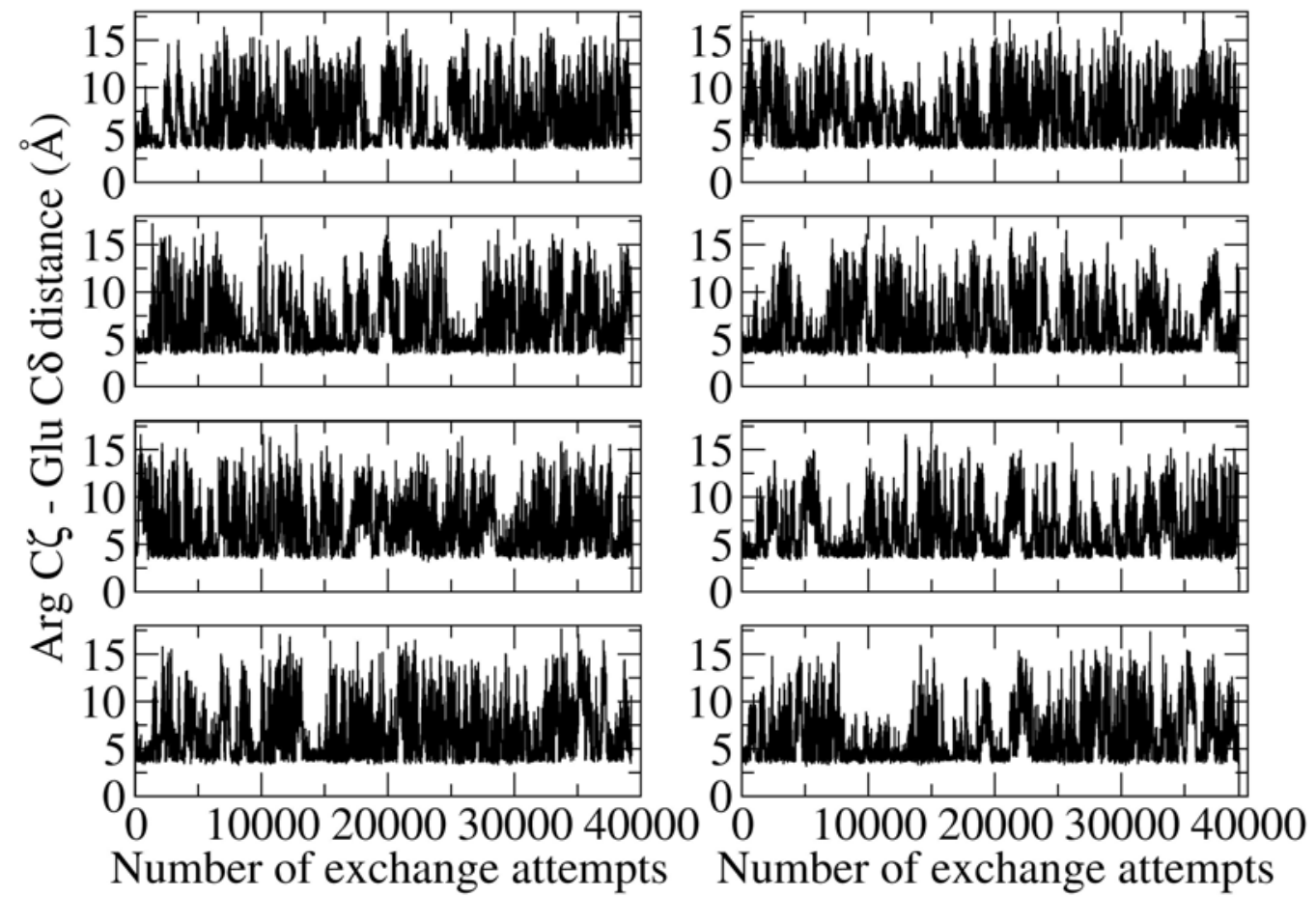

Figure S.2. Distributions of the Arg $\mathrm{C} \zeta$ - Glu C $\delta$ salt bridge distance for 8 replicas for TIP3P REMD simulation. All replicas show multiple salt bridge forming and breaking events. Remaining replicas show similar profiles ensuring converged ensembles. 


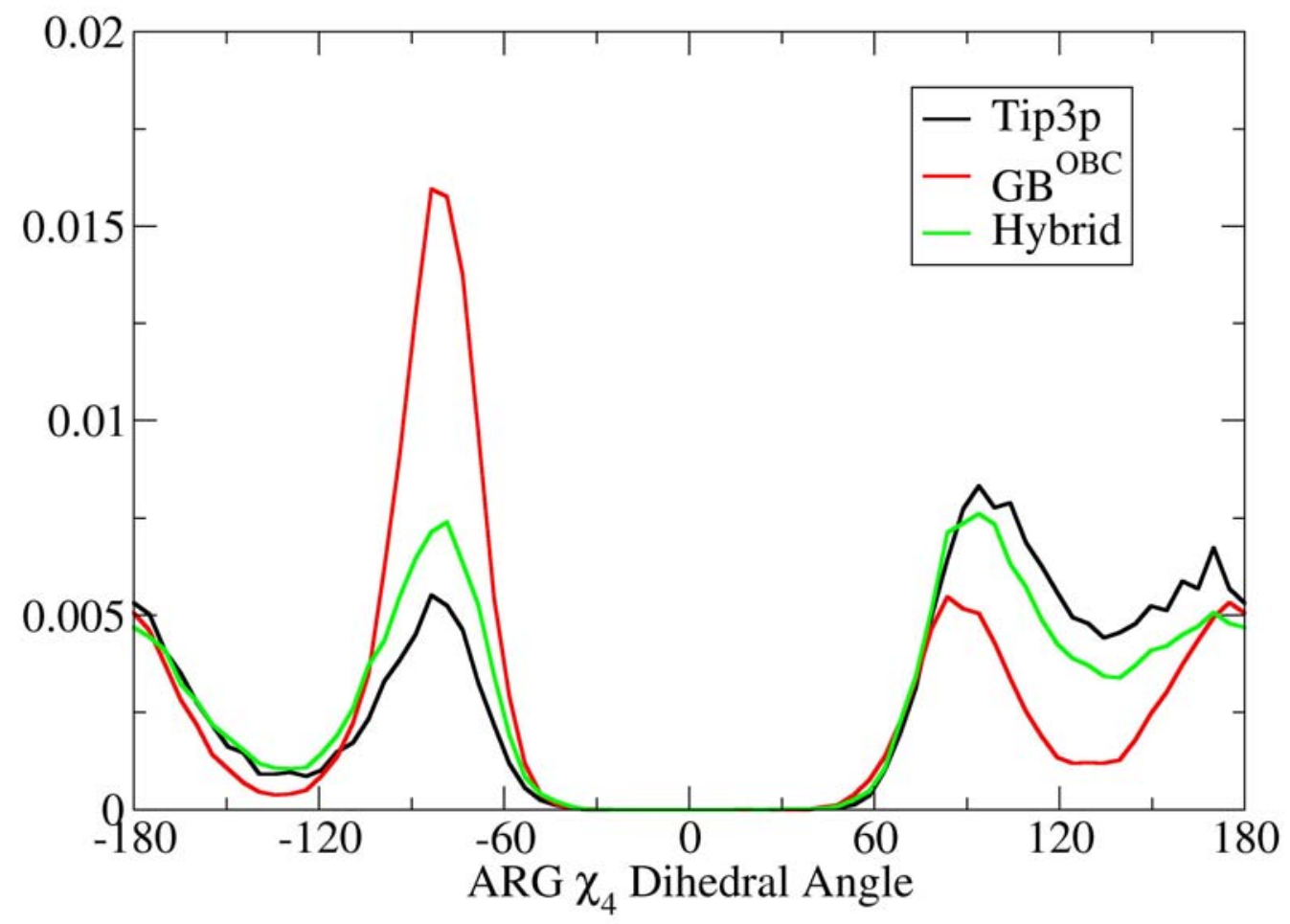

Figure S.3. Distribution of $\chi_{4}$ dihedral angle of the ARG residue for all solvent models. TIP3P and hybrid simulations show similar profile whereas GB simulations prefer one conformation over the other one. This observation is consistent with different side chain orientation observed with both solvent models. 